\title{
The interaction between memorized objects and abrupt onsets in oculomotor capture
}

\author{
Jason H. Wong • Matthew S. Peterson
}

Published online: 2 May 2011

(C) Psychonomic Society, Inc. 2011

\begin{abstract}
Recent evidence has been found for a source of task-irrelevant oculomotor capture (defined as when a salient event draws the eyes away from a primary task) that originates from working memory. An object memorized for a nonsearch task can capture the eyes during search. Here, an experiment was conducted that generated interactions between the presence of a memorized object (a colored disk) with the abrupt onset of a new object during visual search. The goal was to compare memory-driven oculomotor capture to oculomotor capture caused by an abrupt onset. This has implications for saccade programming theories, which have little to say about saccades that are influenced by object working memory. Results showed that memorized objects capture the eyes at nearly the same rate as abrupt onsets. When the abrupt onset and a memorized color coincide in the same object, this combination leads to even greater oculomotor capture. Finally, latencies support the competitive integration model: Shorter saccade latencies were found when the memorized color combined with the onset captured the eyes, as compared to either color or onset only. Longer latencies were also found when the color and onset occurred in the same display but were spatially separated.
\end{abstract}

Keywords Oculomotor capture - Visual object working memory $\cdot$ Abrupt onsets $\cdot$ Saccade programming

\footnotetext{
J. H. Wong $\cdot$ M. S. Peterson

George Mason University,

4400 University Dr MS3F5,

Fairfax, VA 22030, USA

Present Address:

J. H. Wong $(\square)$

Naval Undersea Warfare Center,

1176 Howell Street, B1171/2,

Newport, RI 02841, USA

e-mail: wongjasonh@gmail.com
}

Research has shown a strong link between visual attention, eye movements, and visual working memory. Specifically, it has been shown that the contents of visual working memory can affect eye movements, even at an early stage of saccade programming (Mannan, Kennard, Potter, Pan, \& Soto, 2010; Olivers, Meijer, \& Theeuwes, 2006; Soto, Heinke, Humphreys, \& Blanco, 2005; but see Woodman \& Luck, 2007). In many of these studies, the item held in working memory is irrelevant to the task that requires eye movements. In a typical study, a colored disk is held in memory while a visual search task is completed during the memory retention interval. If what is stored in working memory appears in the visual search display, the eyes will often saccade to this stimulus, even if it is completely irrelevant to the task goal of finding a target (but see Houtkamp \& Roelfsema, 2006).

These results suggest that active rehearsal in visual working memory is able to affect early saccade programming processes, possibly due to reentrant processes from memory onto early visual areas of the brain (Zhang \& Luck, 2009). Other studies have shown that irrelevant visual stimuli, such as salient events like sudden onsets, can directly affect saccade programming from the bottom up (Theeuwes, Kramer, Hahn, \& Irwin, 1998). In these cases, when oculomotor capture occurs, it is assumed that the saccadic signals generated by these transient events are strong enough to draw saccades to their locations, overpowering the signal generated by the search target.

Task goals (such as locating a search target), on the other hand, produce a top-down effect on the saccade map. Another factor that influences saccade programming in a top-down fashion is the attentional set, which is the attentional control settings that preferentially weight certain features and can attenuate capture of the visual system. For example, when searching for a color singleton target (e.g., a green circle among gray circles), a shape singleton onset (e.g., the sudden 
appearance of a gray square) may not capture attention strongly, since its features do not match those of the attentional set (Folk, Remington, \& Johnston, 1992). These top-down factors likely play a major role in real-world visual search (Chen \& Zelinsky, 2006) and in the programming of saccades. There is some debate as to whether memorydriven oculomotor capture can be thought of as a top-down or bottom-up process (Theeuwes, 2010), since working memory utilizes frontal brain areas (Goldman-Rakic, 1987) but has a rapid effect on saccade programming (Mannan et al., 2010).

Apart from the top-down or bottom-up nature of memorydriven oculomotor capture, the fact that working memory can capture the eyes so strongly has a direct effect on theories of saccade programming. All theories center on how different signals compete for a saccade. Older theories, such as the independent horse-race theory of Theeuwes (Theeuwes et al., 1998; Theeuwes, Kramer, Hahn, Irwin, \& Zelinsky, 1999) suggested parallel programming of two separate saccades: one, goal-directed saccade intended for a search target, and one, saliency-driven saccade toward the irrelevant singleton. In this model, the first saccade to finish its programming is executed. However, this theory was contrary to evidence that multiple saccade targets interacted in their competition for the first saccade (e.g., the center-of-gravity effect; Coren \& Hoenig, 1972; Findlay, 1982) and has been superseded by theories that account for this spatial interaction.

One such theory is that of competitive integration (Godijn \& Theeuwes, 2002), which hypothesizes a single retinotopic saccade map that is used for the programming of both top-down and bottom-up saccades. Competitive integration states that when a saccade is programmed, areas surrounding that location are excited and distant locations are inhibited. Therefore, two relatively distant saccade targets will inhibit one another (so only one saccade target will be activated), but two relatively close saccade targets will combine their activations, leading to the strongest activation directly between the targets. This model is similar to others that have been previously proposed (Trappenberg, Dorris, Munoz, \& Klein, 2001) and is supported through experimentation (Ludwig \& Gilchrist, 2003; van Zoest, Donk, \& Theeuwes, 2004).

One shortfall of the competitive integration theory is that it focuses on the strength of saccadic signals and only takes signal timing into account at a very broad level, stating that the endogenous signals are processed more slowly than exogenous ones. While the signal generated by a target may be strong, this top-down task goal signal is likely slow to affect the saccade map (as suggested by long saccade latencies to search targets). Similarly, signals generated by bottom-up salient stimuli are weak - as evidenced by that fact that when they are present during search, the eyes are still more likely to go to the goal-directed target - but they are able to affect the saccade map early and can therefore capture the eyes before the target signal is generated. Stimuli stored in working memory also appear to affect the saccade map at an early state, due to their short saccade latencies (Mannan et al., 2010), but not much else is known about the memory-driven oculomotor capture phenomenon.

The experiment described here was designed to explore how the memory-driven oculomotor capture signal interacts with the saliency-driven signal generated by the abrupt onset of a new object. Abrupt onsets were chosen because they generate saliency-driven oculomotor capture signals that are fairly resistant to top-down cognitive control. A working memory retention and recognition task was used here, with a visual search task occupying the memory retention period. The goal was to examine the interactions between the search target, an abrupt onset, and a memorized or nonmemorized color singleton during visual search in competition for the initial saccade.

Different experimental conditions were created in order to determine a baseline rate of oculomotor capture for abrupt onsets and memorized colors (unique colors were included as a control condition). The critical findings of Mannan et al. (2010) were also replicated by combining the abrupt onset and the memorized color singleton. Finally, competition conditions were created by including the search target, memorized color, and abrupt onset in the same search display, but spatially separated. Examining how these objects compete for the initial saccade in the search task will shed light on the memory-driven saccadic signal as well as influencing saccade programming theories themselves in terms of understanding how both the strength and timing of saccade signals affect eye movement programming.

\section{Method}

There were two between-subjects groups due to the number of relevant stimulus configurations (which will be detailed later). In one condition, the color singleton was present during search, and it matched the color used in the memory task (memorized-color condition). In the other condition, the color present during search was a nonmemorized unique color (unique-color condition). Within each group, there were multiple stimulus configurations designed to examine how combinations of different singleton events can capture the eyes. By examining where the eyes first landed during search in these conditions, knowledge will be gained about the properties of the memory-driven signal generated by the memorized color.

\section{Participants}

A total of 30 George Mason University undergraduates participated (6 males, 24 females, average age 20.4 years), 
with 15 participants randomly assigned to each betweensubjects group. The naïve observers received partial class credit in exchange for their participation, and all had normal or corrected-to-normal color vision.

Apparatus and stimuli

A Mac Pro equipped with a 21 -in. (20-in. viewable) monitor operating at $85 \mathrm{~Hz}$ with a resolution of $1,024 \times 768$ was used to display stimuli. This computer was networked to a Dell Pentium 4 that collected eyetracking data in conjunction with an EyeLink 2 eyetracker (SR Research). The EyeLink 2 system samples at a rate of $250 \mathrm{~Hz}$ and has spatial resolution of $0.2^{\circ}$. The head was stabilized by means of a chinrest located $70 \mathrm{~cm}$ from the monitor.

The memory stimuli consisted of a colored disk that was $3.90^{\circ}$ in diameter, centrally located on the screen. Colors were chosen from four broad groups: red, blue, green, and yellow. Eight different variations of each color group were used, creating a total of 32 colors in the set.

Colors were chosen to be similar, yet not too difficult to discern from each other. The colors were also chosen to be as equiluminant as possible, and since all analyses were performed by collapsing across all colors, differences in luminance should have averaged out across participants.

Between the presentation of the memory stimuli and the search task, a search preview screen was displayed (see Fig. 1) in order to establish search object locations so that a new object could be abruptly onset when the actual search objects were shown. This display consisted of six square-circle hybrid masks that were designed as a combination of the target square and distractor circle shapes. This was done to minimize visual noise (and possible attenuation of attention capture) when transitioning from the preview screen to the search display (Martin-Emerson \& Kramer, 1997).

For the search task, the distractors were gray disks measuring $3.90^{\circ}$ in diameter, and the target was a gray square of the same size. Each search display consisted of six objects: five distractors and one target. The shapes were located $9.33^{\circ}$ away from the center of the screen, arranged around an invisible circle with $60^{\circ}$ of angular distance between each object. Within each search distractor, an upor down-facing $\mathrm{C}$ was present. Within the search target, a left- or right-facing $\mathrm{C}$ was present. These figures were colored black, centered within each object, and $0.03^{\circ}$ by $0.03^{\circ}$ in size. Therefore, these objects were too small to be discriminated by peripheral vision and required direct fixation to be identified.

\section{Design and procedure}

This experiment contained one between-subjects manipulation: whether the color singleton in search (if present) matched the memorized color (memorized-color group) or was a unique color (unique-color group). Within each of those two between-subjects conditions, there were seven within-subjects stimulus configuration conditions corresponding to various control, cooperation, or competition search conditions. Examples of these seven conditions can be seen in Fig. 2.

The first stimulus configuration condition was a baseline condition that did not contain any singletons (none condition). Additional baseline conditions were those in which only an abrupt onset was present (onset-only condition) or in which a single distractor (not abruptly onset) was not gray, but instead a unique or memorized color (color-only condition). One condition included the abrupt onset of a unique or memorized color, thus combining the onset and color singletons into a single object (combined condition). Finally, three conditions included both an abrupt onset and a separate color singleton in the same display. These two singletons could appear in the display separated by three angular distances $\left(30^{\circ}, 90^{\circ}\right.$, or $150^{\circ}$ ) leading to three separate conditions (apart-30, apart-90, and apart-150). These three conditions created

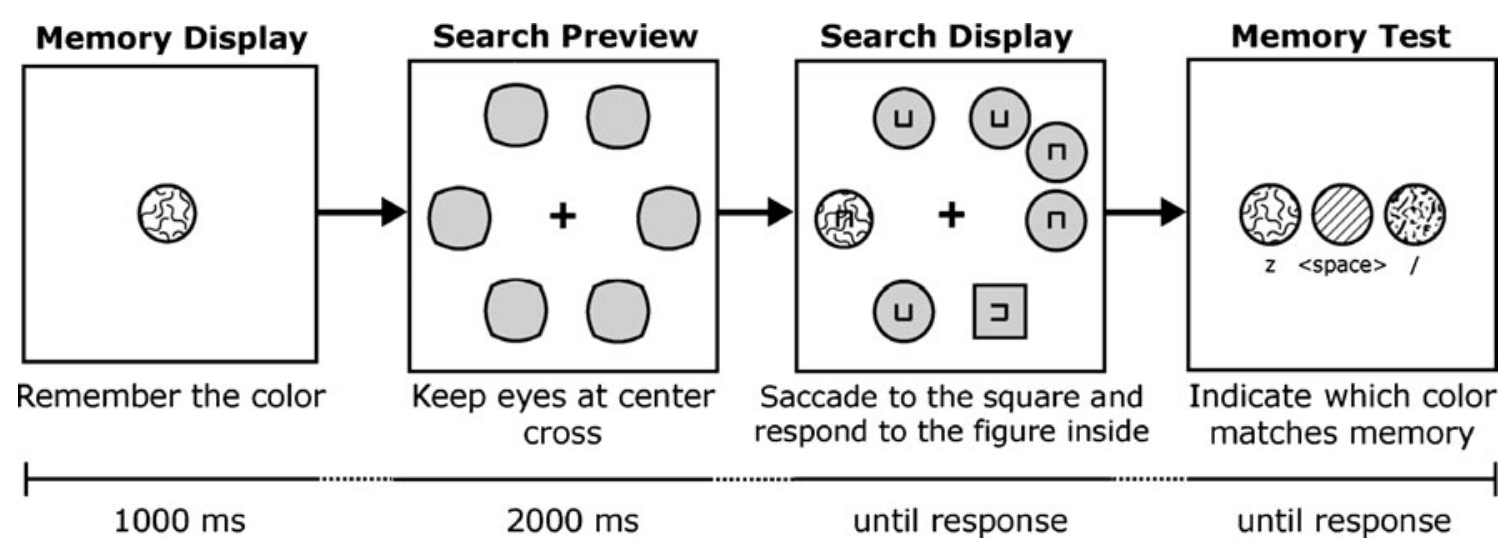

Fig. 1 Trial schematics for this experiment. In this black-and-white image, colors are represented by different patterns 

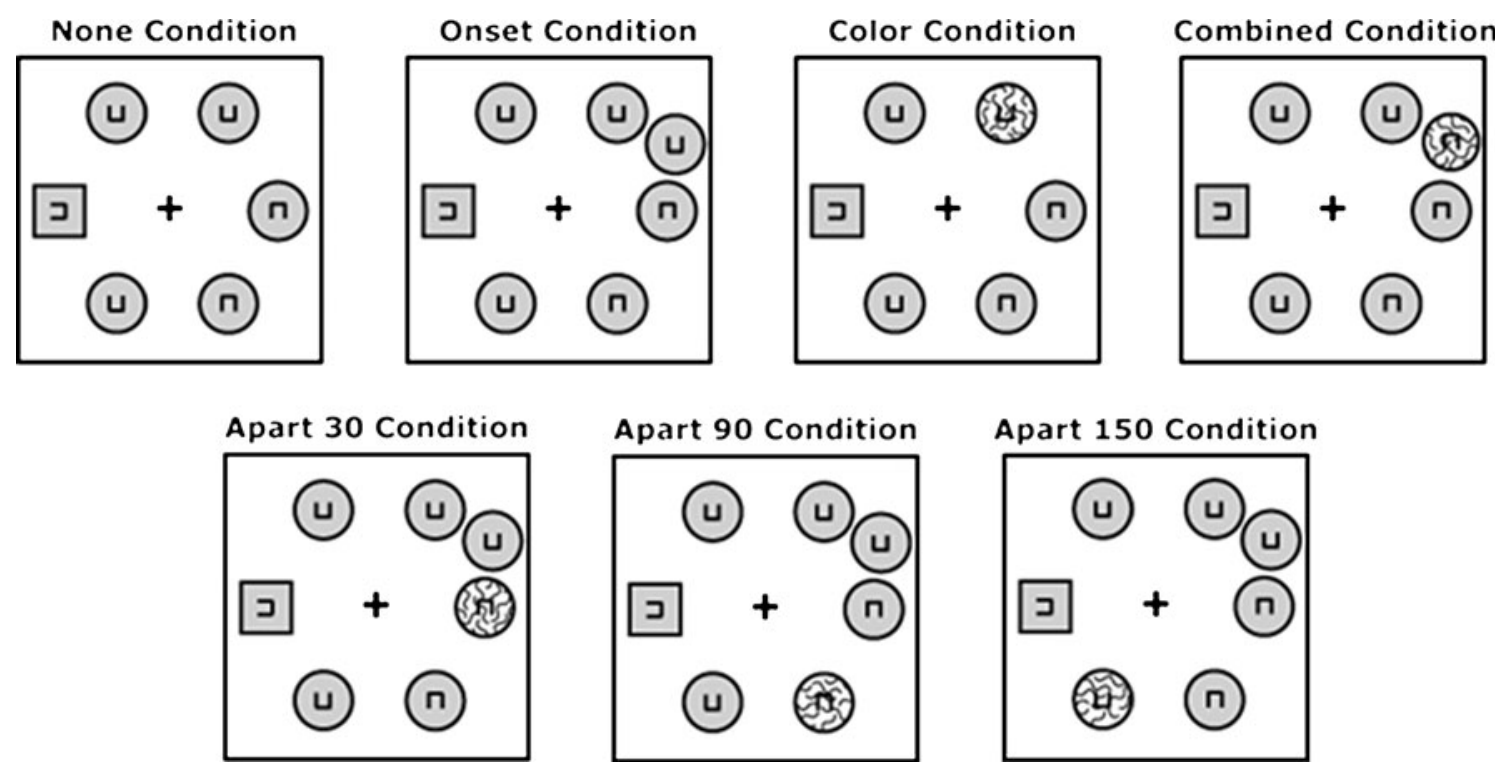

Fig. 2 Example stimulus configurations of each of the seven conditions. The conditions in the top row are used to examine cooperation between the abrupt onset and memorized color (shown in a different fill pattern),

competition between the onset and color singletons for the initial fixation.

Each participant was randomly assigned to one betweensubjects condition and then received 40 trials each of the seven stimulus configuration manipulations. This led to a total of 280 trials. Within each condition, the colors used for the memory task, the location of the target during search, and the figure present within the search target were randomized. Participants saw trials that were very similar to the one depicted in Fig. 1. The memory display contained a colored disk at the center of the screen that was presented for $1,000 \mathrm{~ms}$. The memorized color was chosen randomly among the four color groups. Participants were told to remember this color in as visual a manner as possible. After this display, the search preview display was presented for 2,000 ms. Participants' eyes were tracked to ensure that the eyes remained at the central fixation cross during this period.

Participants then saw the search display, containing a square target and at least five gray circle distractors. Participants were to make a saccade to the target and respond with a keypress (either "z" or "”") based on the figure within. The system would not accept a response until the eyes were fixated on the target, and if an incorrect response was made, the system beeped. The response keys were counterbalanced across participants.

In conditions containing an onset, another gray circle distractor could appear between the search objects previewed in the previous screen. This onset would be located between two objects. In conditions containing a color singleton, a colored distractor would be present in an already previewed location. In the combined condition, a and the conditions in the bottom row are used to examine competition between the singletons

colored abrupt onset would appear between search objects. Finally, in the competition conditions, both an abrupt onset and a color singleton were present in the display, separated by $30^{\circ}, 90^{\circ}$, or $150^{\circ}$.

After a response was made for search, there was a 500-ms delay before the memory test was presented. Three colored disks were shown, centered in the display. One disk matched the memory item exactly, while the other two were randomly chosen from the same color group (e.g., three red disks of different shades were displayed). This encouraged participants to encode the memorized color in a more visual manner. Participants indicated whether the left, middle, or right colored disc matched their memory. A feedback display was shown, and the next trial began.

\section{Results}

An average of $5.75 \%$ of trials were discarded because participants did not remain fixated at the center of the screen before the onset of the search display. A fixation was determined to be on an object if the eyes were no more than $4.68^{\circ}$ from the center of the object, which was $3.90^{\circ}$ in diameter.

\section{Memory accuracy}

A $2 \times 7$ ANOVA with Experimental Group as a betweensubjects factor and Stimulus Configuration Condition as a within-subject factor was performed on memory accuracy (see Table 1 for the means). The results showed a trend 
Table 1 Memory accuracy (with standard errors in parentheses) for all conditions across both groups

\begin{tabular}{llllllll}
\hline Group & None & Onset & Color & Combined & Apart 30 & Apart 90 & Apart 150 \\
\hline Memorized color & $67.33(3.98)$ & $68.33(3.31)$ & $67.50(2.81)$ & $66.67(2.46)$ & $69.83(3.17)$ & $67.00(2.87)$ & $68.00(2.56)$ \\
Unique color & $75.50(2.28)$ & $74.83(2.54)$ & $73.00(2.18)$ & $73.50(2.34)$ & $73.33(1.93)$ & $72.50(2.26)$ & $69.00(2.69)$ \\
\hline
\end{tabular}

The numbers are expressed as percentages of trials answered correctly

toward a memory accuracy difference across experimental groups $[F(1,28)=3.22, p=.08]$. However, this trend suggests that the unique-color group had greater memory accuracy than the memorized-color group $(M=.68, S E=.02$ for the memorized-color group; $M=.73, S E=.02$ for the unique-color group). There was no effect of stimulus configuration condition $[F(6,168)=0.76, p=.60]$, nor was there an interaction $[F(6,168)=0.81, p=.58]$.

It is reasonable to hypothesize that memory accuracy would increase for trials on which the memorized color was directly fixated during search. However, a $2 \times 5$ withinsubjects ANOVA demonstrated that this was not the case. Memory accuracy data for the memorized color were examined, with First Fixation Destination (target or color singleton) and Stimulus Configuration Condition as factors. There was no interaction $[F(4,56)=1.36, p=.26]$, and more importantly, there was no main effect of the first fixation destination on memory accuracy $[F(1,14)=1.22$, $p=.29]$. Therefore, even when the eyes landed on the color singleton, memory accuracy was not different from when the eyes fixated the target first.

Search accuracy

A $2 \times 7$ ANOVA was conducted on search accuracy. There was an effect of experimental group $[F(1,28)=8.25, p<.01]$ and a trend toward an effect of stimulus configuration, as well $[F(6,168)=2.06, p=.06]$. However, there was no evidence of an interaction $[F(6,168)=1.27, p=.28]$. Although search accuracies across conditions were uniformly high $(M=95.60 \%, S E=1.27$, for the memorizedcolor group; $M=98.79 \%, S E=0.56$, for the unique-color group), the lower accuracy for the memorized-color group might have been due to increased interference from the colormatching singleton.
Search reaction times

The $2 \times 7$ ANOVA performed on search reaction times (Table 2) showed no effect of experimental group $[F(1,28)=0.01, p=.92]$ but did show an effect on stimulus configuration $[F(6,168)=7.73, p<.001]$. There was no interaction between these factors $[F(6,168)=0.92$, $p=.49]$. Orthogonal contrasts demonstrated that search was faster when there were no singletons present (none condition vs. all remaining conditions), $F(1,29)=28.39$, $p<.001$, and when there were one versus two singletons present, $F(1,29)=15.12, p<.01$. This finding supports the idea that search singletons were capturing the eyes, which led to longer target-finding times. This was assessed more directly by examining first fixations.

\section{First fixations}

Cooperation conditions We expected to replicate the findings of Mannan et al. (2010) because their paradigm was similar to the one used in this study. First fixations to singletons were examined across the cooperation conditions in both the memorized-color and unique-color groups. Replication would be achieved if the memorized color was fixated more often than the unique-color singleton. A series of $t$ tests were performed on first fixation data (Fig. 3) to examine fixation rates to the various saccade targets.

By including onset-only and color-only control conditions, the analyses here can go further than those in Mannan et al. (2010). Specifically, capture rates of the combined stimulus were compared against the onset-only and color-only control conditions. To begin this examination, a $2 \times 2 \times 3$ ANOVA was performed with Experimental Group (memorized-color and unique-color) as a between-subjects factor and First

Table 2 Search reaction times (with standard errors in parentheses) for all conditions across both groups

\begin{tabular}{|c|c|c|c|c|c|c|c|}
\hline Group & None & Onset & Color & Combined & Apart 30 & Apart 90 & Apart 150 \\
\hline Memorized color & $913.20(68.34)$ & $959.50(54.23)$ & $956.61(65.29)$ & $983.75(61.78)$ & $997.29(67.19)$ & $1,023.35(73.15)$ & $1,034.10(77.99)$ \\
\hline Unique color & $934.09(39.01)$ & $1010.61(34.86)$ & $974.70(34.20)$ & $975.38(35.74)$ & 992.11 (39.67) & $1,015.98(37.89)$ & $1,018.01(37.52)$ \\
\hline
\end{tabular}


Fig. 3 Two graphs representing the first fixations to either the target or the singleton in the cooperation conditions. The data are grouped by the type of singleton present in search. The combined stimulus captured the eyes at a greater rate in the memorized-color group than did either the onset or the memorized color alone
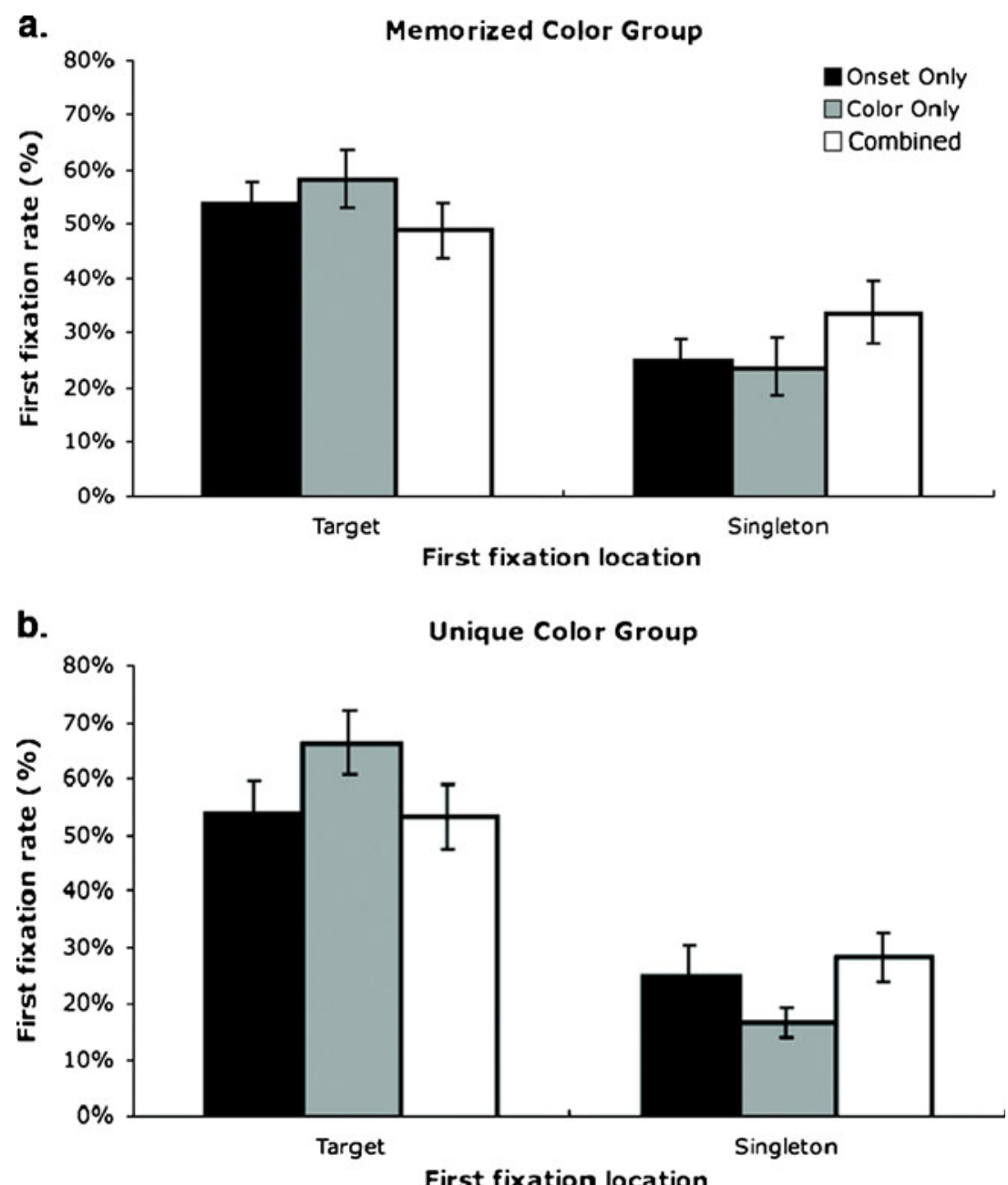

First fixation location

Fixation Destination (target or singleton) and Stimulus Configuration Condition (onset-only, color-only, or combined) as within-subject factors.

The ANOVA showed no main effect of experimental group $[F(1,28)=0.00, p=.98]$ or of stimulus configuration $[F(2,56)=2.31, p=.10]$ but did show a main effect of first fixation destination $[F(1,28)=23.62$, $p<.001$ ], with the eyes being more likely to go to the target. The Experimental Group $\times$ Stimulus Configuration and Experimental Group $\times$ First Fixation Destination interactions were not significant $[F(2,56)=0.13, p=.88$, and $F(1,28)=0.45, p=.51$, respectively], but there was a significant interaction between stimulus configuration and first fixation destination $[F(2,56)=15.41, p<.001]$. Finally, there was no three-way interaction between these factors $[F(2,56)=1.84, p=.17]$.

Linear contrasts were performed between specific conditions in the memorized-color group (Fig. 3a). Contrasts showed no difference between the first fixation rates to the onset $(M=24.76 \%, S E=3.91)$ and to the memorized color $(M=23.61 \%, S E=5.28), F(1,14)=0.08, p=.79$, so both singletons captured the eyes at similar rates. Another contrast examining fixations to the combined singleton showed that the combined stimulus captured the eyes at a greater rate than that shown by the collapsed onset and color singletons $(M=33.60 \%, S E=5.76), F(1,14)=6.20$, $p<.05$. This suggests similar signal strength between the color and onset singletons, and increased strength when they are combined.

Similar analyses were performed on the unique-color experimental group (Fig. 3b). Here, the abrupt onset captured the eyes more often than did the color singleton $(M=24.88 \%, S E=5.13$, for the onset; $M=16.55 \%$, $S E=2.73$, for the color), $F(1,14)=5.35, p<.05$. No cooperation was found in this group; contrasts showed no difference in the rates at which the onset and combined singletons captured the eyes $[F(1,14)=0.82, p=.37]$, and these rates were greater than that of the unique color alone $[F(1,14)=10.21, p<.01]$. Therefore, the color singleton seemed to contribute nothing to the oculomotor capture ability of the combined singleton.

To determine whether or not memorizing the color increased capture relative to the onset baseline, difference scores (color minus onset) were calculated for each group. 
In order to minimize boundary effects, the fixation rates were logit transformed before subtraction (data from 1 participant were excluded because he had a capture rate of 0 , and the logit transform of 0 is undefined). The memorized-color condition led to significantly more capture than the unique color condition, relative to the onset $[t(27)=1.88, p<.05]$, demonstrating that the memory condition was able to significantly increase capture by an irrelevant color.

Competition conditions Analyses were conducted to examine the interaction between abrupt onsets, color singletons, and targets when they were spatially separated by $30^{\circ}, 90^{\circ}$, and $150^{\circ}$. A $2 \times 3 \times 3$ ANOVA was performed with Experimental Group as a between-subjects factor and First Fixation Destination (target, onset, and color singleton) and Stimulus Configuration (apart-30, apart-90, and apart-150) as within-subjects factors.

The ANOVA on the first fixation data (Fig. 4) showed no main effect of experimental group $[F(1,28)=0.29$, $p=.59]$ or of stimulus configuration $[F(2,56)=0.31$, $p=.73]$, but did show a main effect of first fixation destination $[F(2,56)=44.48, p<.001]$. Additionally, there was no interaction between experimental group and stimulus configuration $[F(2,56)=0.38, p=.69]$. An interaction between experimental group and first fixation destination was also not seen $[F(2,56)=2.01, p=.14]$. There was an interaction between stimulus configuration and first fixation destination $[F(2,56)=2.44, p<.05]$, but no three-way interaction $[F(2,56)=0.42, p=.79]$.

Linear contrasts were used to determine whether capture rates were significantly different between the color singleton and the abrupt onset (Fig. 4a). The memorized-color singleton captured the eyes more often than did the onset [ $M=19.90 \%$ for the memorized color, $M=13.81 \%$ for the abrupt onset; $F(1,56)=18.5, p<.01]$. For the uniquecolor condition, the results were reversed, with the onset ( $M=16.14 \%$ ) capturing the eyes more often than did the unique-color singleton $(M=11.27 \%)[F(1,56)=6.36$, $p<.05]$.

In both the memorized-color and unique-color groups, the distance between the onset and color had no effect on first fixations to the singletons. This is suggested by orthogonal contrasts between the apart-30 condition and the apart-90 and
Fig. 4 Two graphs representing the first fixations to the target, color singleton, or abrupt onset in the memorized- and uniquecolor groups, organized by distance between the color and onset singletons. The memorized color often captured the eyes more than the onset, but the unique color did not
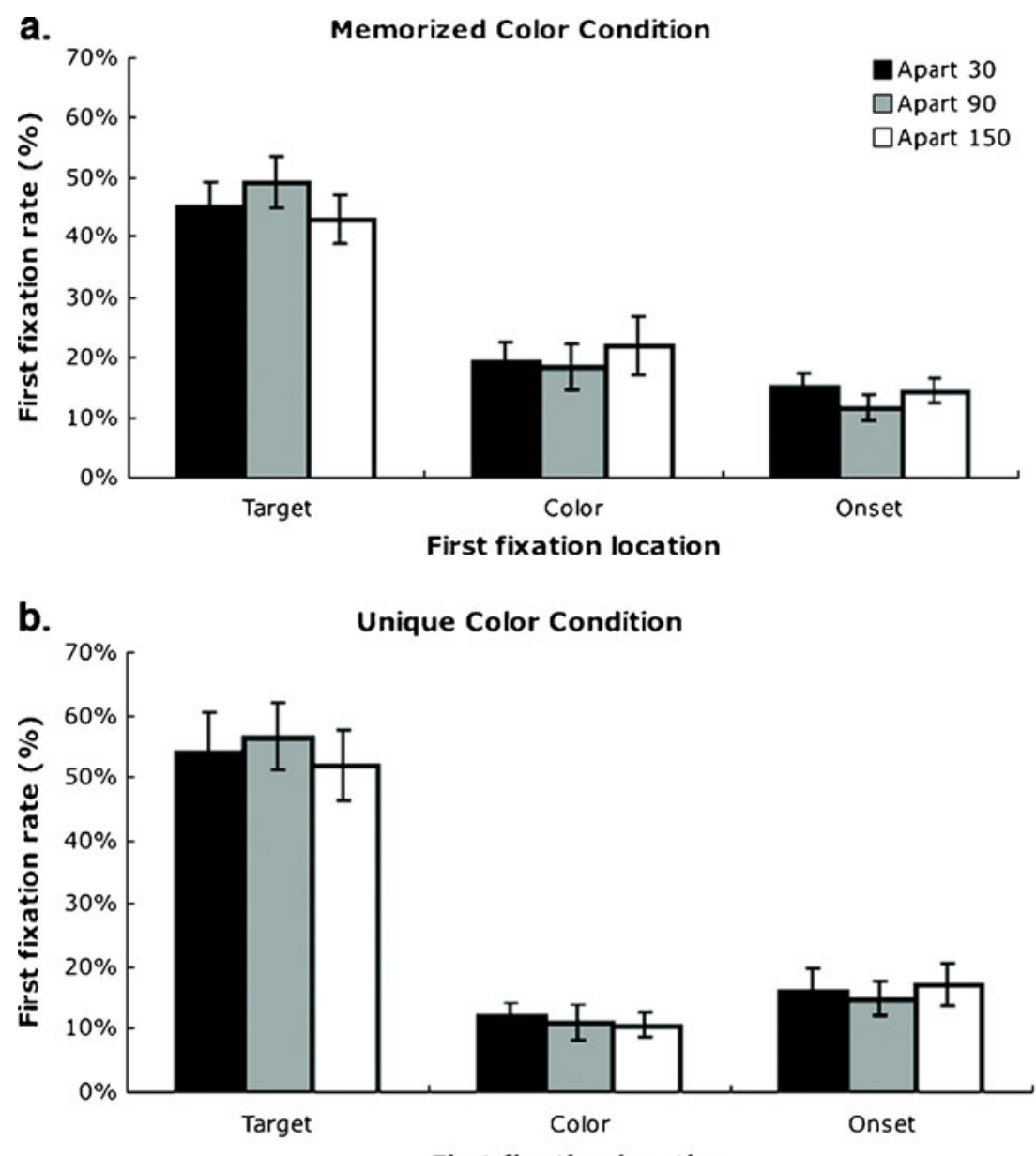

First fixation location 
apart-150 conditions together $[F(1,14)=0.14, p=.71$, for the memorized-color group; $F(1,14)=0.22, p=.64$, for the unique-color group] and between the apart-90 and apart-150 conditions $[F(1,14)=2.31, p=.13$, for the memorized-color group; $F(1,14)=0.01, p=.94$, for the unique-color group].

Distance from target By examining fixations on an abrupt-onset or memorized-color singleton as a function of their distance from the target (by averaging across first fixation data in the apart-30, apart-90, and apart-150 conditions), the influence of the strong, goal-driven signal on saccade programming can be determined (Fig. 5).

A $2 \times 3$ ANOVA with Singleton Type (onset and memorized color) and Target Distance (near, middle, and far) as factors was conducted. For onsets, the near, middle, and far distances were $30^{\circ}, 90^{\circ}$, and $150^{\circ}$ away from the target, respectively. For memorized colors, these distances were $60^{\circ}, 120^{\circ}$, and $180^{\circ}$ away from the target. The results indicated no main effect of the singleton type $[F(1,14)=1.72, p=.21]$, but there was a main effect of target distance $[F(2,28)=5.53, p<.01]$ and an interaction between the two factors $[F(2,28)=4.19, p<.05]$.

Further examination of distance effects were conducted using orthogonal contrasts, and while an effect was found for onsets near the target $(M=20.41 \%, S E=2.92)$ versus in the middle or far distances $[F(1,14)=22.18, p<.001]$, no effect was found between the middle and far distances $(M=9.71 \%, S E=1.60$, for middle distances; $M=12.09 \%$, $S E=2.41$, for far distances) $[F(1,14)=1.28, p=.27]$. No effect of the memorized-color distance from the target was found ( $F<1$ for all distance comparisons). Overall, the onset received more first fixations if it was near the target; otherwise, the target had little effect on first fixations to onsets at farther distances or on memorized-color singletons at any distance.

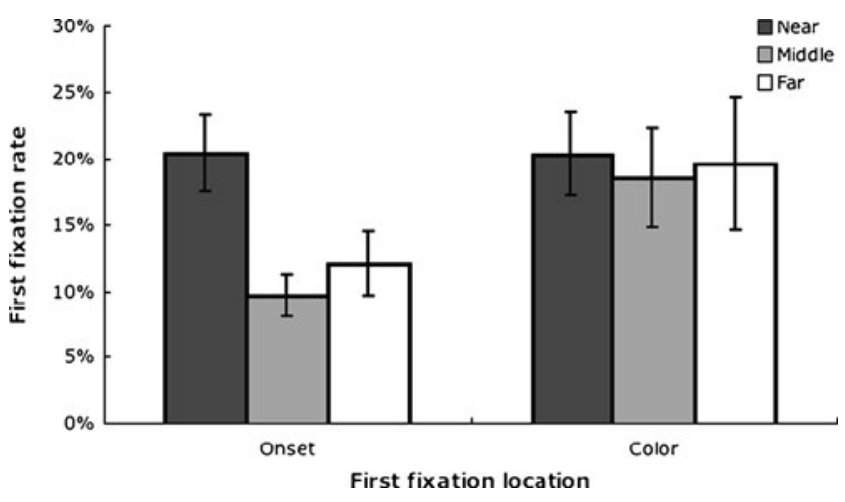

Fig. 5 First fixation rates based on the location of the first fixation, grouped by distance between the singleton and the target (near, middle, and far distances). This graph represents data from the memorized-color group only. There is an effect of distance from the target for onsets but not for memorized-color singletons
Singleton-saccade latencies

It is possible for saccade latencies to be affected by working memory as well. A $2 \times 3$ ANOVA was conducted on saccade latencies to search singletons with Experimental Group (memorized-color and unique-color) as a between-subjects factor and Saccadic Destination (target, onset, and color) averaged across stimulus configuration conditions as a within-subject factor (see Table 3 for the means).

Analyses showed a main effect of saccadic destination $[F(2,50)=67.34, p<.001]$, no main effect of experimental group $[F(1,25)=0.15, p=.70]$, and no interaction $[F(2,50)=0.62, p=.54]$. Latencies to the target were slowest when compared to latencies to the onset or color for both the memorized-color group $[F(1,14)=41.60, p<.001]$ and the unique-color group $[F(1,14)=122.48, p<.001]$. Additionally, latencies were faster to the color singleton than to the abrupt onset. This effect was significant in the memorized-color group $[F(1,14)=5.43, p<.05]$ and a trend in the unique-color group $[F(1,14)=3.37, p=.08]$, suggesting that the color singleton caused a saccade to be executed more quickly than a saccade to the onset.

A series of planned comparisons was performed to test the horse-race and competitive integration models. According to both models, when the onset and singleton occur in the same location, shorter saccade latencies to the onset or color singleton should occur, as compared to when they occur alone or when they occur together but in different locations. In the case of the horse-race model, two signals occurring at the same time and the same location will increase the likelihood of a fast saccadic reaction time to that location (Miller, 1986), whereas for the competitive integration model, the integration of the two signals would lead to shorter saccade latencies. For the memorized-color group, saccade latencies were faster to the combined stimulus $(M=277 \mathrm{~ms})$ than to the onset alone $(M=318 \mathrm{~ms})[t(11)=1.9, p<.05]$ and to the color singleton alone $(M=304 \mathrm{~ms})[t(11)=2.4, p<.05]$. For the unique group, saccade latencies to the combined stimulus $(M=265)$ were faster only than those to the onset when it was alone $(M=288 \mathrm{~ms})[t(12)=2.5, p<.05]$. The lack of a difference between the latencies to the color alone and the combined stimulus $[t(12)<1]$ might have been due to a floor effect.

Table 3 Saccade latencies (with standard errors in parentheses) for search stimuli across both groups

\begin{tabular}{llll}
\hline Group & Target & Onset & Color \\
\hline Memorized color & $397.15(23.71)$ & $325.42(16.27)$ & $287.62(9.27)$ \\
Unique color & $396.67(14.85)$ & $305.67(11.59)$ & $286.39(9.43)$ \\
\hline
\end{tabular}


Unlike the capture rates, these findings support both the horse-race and competitive integration models. One difference between the horse-race and competitive integration models is that the competitive integration model predicts that (1) increases in distance between the two competing stimuli should lead to slower saccadic reaction times then when the stimuli are combined, and (2) this effect should be largest at the greatest distance. Figure 6 shows the saccade latencies to the onset and target broken down by these conditions.

To test for this pattern, $t$ tests were performed comparing the saccade latencies at $30^{\circ}$ and $90^{\circ}$ separation, as well as those at $90^{\circ}$ and $150^{\circ}$, for saccades that went to an onset or to a color singleton for the two groups. For the memorizedcolor groups, saccades to the onset were significantly slower when it was $90^{\circ}$ away from the color singleton $(M=341 \mathrm{~ms})$ than when it was $30^{\circ}$ away from the color singleton $(M=$ $311 \mathrm{~ms})[t(11)=3.7, p<.01]$, and significantly slower again when the onset was $150^{\circ}$ away from the color than when it was $90^{\circ}$ away $(M=375 \mathrm{~ms})[t(11)=2.3, p<.05]$. Additionally, saccades to the onset were slower when a color singleton was $150^{\circ}$ away than when the onset occurred by itself $(M=318 \mathrm{~ms})[t(11)=2.6, p<.05]$. However, this effect was asymmetrical for the memorized-color group, with the onset having no effect on latencies to the color singleton. In summary, the distance effects for the saccade latencies do not support the horse-race model and instead are in line with the competitive integration model.

For the unique-color group, the distance effect was limited to $30^{\circ}$ versus $90^{\circ}$, and this effect was for latencies both to the onset $[M=277$ and $330 \mathrm{~ms} ; t(10)=2.6, p<.05]$ and to the color singleton $[M=274$ and $304 \mathrm{~ms} ; t(10)=2.4, p<.05]$. Likewise, saccade latencies to the onset and to the color were slower at the extreme distances as compared to when the onset or color singleton occurred alone [lowest $t(11)=2.37$, $p<.05]$. Again, this pattern of results goes against the horserace model and supports the competitive integration model.
Fig. 6 Saccade latencies to the onset or the color singleton. The top graph is from the memorizedcolor group, and the bottom is from the unique-color group. The "apart" condition represents the combined data for when the onset and color singleton were $30^{\circ}, 90^{\circ}$, and $150^{\circ}$ apart. For the combined condition, the onset and color singletons occurred in the same location, and thus the two bars represent the same data. In the memorized-color condition, latencies to the onset were significantly affected by the distance of the color singleton, and the most extreme distance led to slower latencies than when the onset occurred alone. Identical results were found for the saccade latencies to the onset and the color singleton for the unique-color group. Brackets indicate that the "Apart" condition is a composite of the apart- $30^{\circ},-90^{\circ}$, and $-150^{\circ}$ conditions
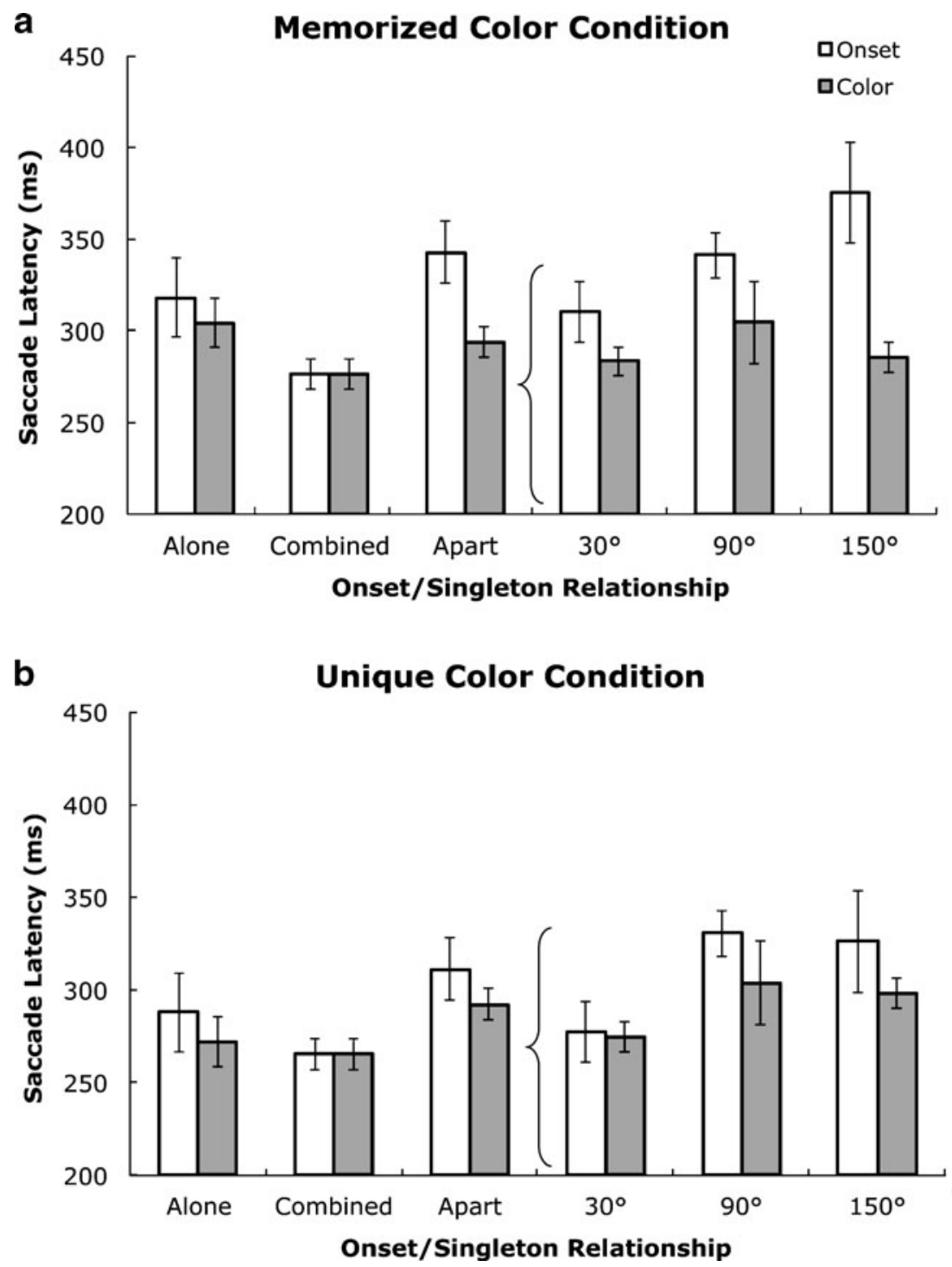
Target saccade latencies

Saccade latencies to the target as a function of distance from the target to the onset or color singleton for each group were compared using separate $2 \times 3$ repeated measures ANOVAs with Distractor Object (onset or singleton) and Distance of Distractor From the Target (near, middle, or far) as factors. Neither object affected saccade latencies to the target (lowest $p=.15$ )

\section{Discussion}

The goal of this experiment was to pair abrupt-onset singletons with memorized-color singletons to examine how they interact when they cooperate or compete for the initial fixation during search. The properties of the memory-driven signal are relatively unknown, and it was not obvious how this signal would interact with the saliency-driven signal of an abrupt onset.

These signals cooperated to capture the eyes when the memorized color and the abrupt onset were associated with the same object, and thus were in the same location in the search display. Since the capture rate increased when the singleton matched the memorized color in the cooperation condition, this suggests that the signals generated by the onset and the memorized color combined their strength to drive the eyes. More specifically, when these signals combined, they captured the eyes at a greater rate than either the memorized color or the onset alone. This did not occur when the onset was paired with a unique, nonmemorized color. The onset alone captured the eyes as much as the onset plus unique color, suggesting that the unique color did not add to the onset signal's ability to capture the eyes.

All participants were required to remember a color during every trial; thus, there were no trials on which object working memory was not occupied. Despite the lack of a memoryless control condition, there is still reason to believe that active memory rehearsal was driving the elevated capture effect. Olivers et al. (2006) conducted a series of studies with a paradigm similar to the one used here and, in one study, reordered the trial sequence. In their Experiment 5, participants first memorized a color and then were tested on it before the search task. This sequence of events did not require rehearsal for the memorized color during search, and the results demonstrated that the presence of the memorized color no longer captured attention. These results suggest that, when the memorized color no longer has behavioral relevance, it does not draw the eyes involuntarily.

While there is evidence that the combination of the abrupt onset and memorized color in the combined stimulus increases the rate at which oculomotor capture occurs, this effect is not additive in nature. If these two stimuli were additive when combined, the eyes should have been captured by the combined stimulus at a rate equal to the sum of the rates of the onset and the memorized color alone (approximately 48\%). Instead, the combined stimulus captured the eyes $34 \%$ of the time. Therefore, while these signals presumably combine their activations in the saccade map, the resulting excitation was not strong enough to capture the eyes at an additive rate; instead, there was some overlap or other attenuating factor against full additivity.

This experiment also contained stimulus configurations designed to put the saliency-driven onset signal in competition with the memory-driven color signal. Evidence from these conditions further demonstrates the strength of the memorized-color singleton as compared to the abrupt onset. Independent of the distance between the onset and the memorized color, the memorized color captured the eyes more often than the onset. However, the target received the greatest number of first fixations, which is not surprising, given previous findings (Theeuwes et al., 1998). What is surprising is that the distance between the target and any singleton had little effect on first fixations on the singleton (with one exception: when the onset was directly next to the target). This suggests that the goaldriven saccade signal generated by the target did not combine its activation with any singletons. It is possible that this was due to the timing of the generation of the individual signals. Saccade latencies have demonstrated that a saccade to the target is executed later than a saccade to an onset or color, indicating that the target signal develops more slowly. Due to this, the timing difference may hinder the ability of the target to dramatically affect saccades to other locations.

These results can be compared to those for the uniquecolor group, for whom the onset and unique color captured the eyes at the same rates when in competition for the first saccade, except in the condition in which the two stimuli were the farthest apart (in that case, the onset captured the eyes at the greatest rate). This result was unexpected, because the onset in the onset-only baseline condition captured the eyes at a significantly greater rate than the unique color did in the color-only baseline condition, which would suggest a stronger onset signal. It is possible that the appearance of the abrupt onset in these competition conditions led to a generalized loss of control over the eyes, which made them susceptible to capture by any singleton, but this hypothesis requires further testing.

One final result that speaks to how quickly the memorized-color singleton generates a signal are the saccade latencies to the target, onset, and color stimuli. Saccades made to the target led to the longest latencies, reflecting the complex computations required to plan a 
goal-driven saccade. Saccades made to the onset and the unique color had much shorter latencies, indicating the involuntary nature of such exogenous saccades. Eye movements to the memorized color, when compared to onsets in the same condition, led to the shortest saccade latencies. This effect was small (about $40 \mathrm{~ms}$ ) but significant, and it suggests that object memory is able to influence saccade programming at a very early stage (Kirchner, Barbeau, Thorpe, Régis, \& Liégeois-Chauvel, 2009).

The general finding that the memory-driven signal is on par in terms of strength with the saliency-driven abrupt onset signal has implications for saccade programming models. While influential theories such as the competitive integration model suggest that objects generate a strong excitatory signal at nearby locations and inhibitory signals at distant locations, this was not supported by the capture data, in which the distance between the onset, color, and target stimuli rarely had an effect on the location of the first fixation: This only occurred when the onset and target were $30^{\circ}$ apart along an imaginary circle.

The independent horse-race model (Theeuwes et al., 1998), however, does take into account signal timing. This model states that saccades are programmed independently, and the first one to be completed is executed. However, this model does not account for the strength of activations by each relevant search object or some form of top-down control. The data show that the target, rather than other salient objects, receives the majority of first fixations, yet displays longer saccade latencies. Therefore, the speed at which information can affect saccade programming cannot be the only factor affecting the winner-take-all activations on the saccade map. The target likely generates a very strong signal, but it affects the saccade map late. This suggests that when an onset or color singleton generates a signal, the signal is generated quickly, yet it is not always sufficiently strong to lead to capture. Alternatively, some form of top-down saccadic inhibition could also be playing a role, which could begin during the search preview screen and keep the eyes at fixation until the target is localized, suppressing fixations to nontarget objects. This is consistent with the cognitivecontrol hypotheses of Woodman and Luck (2007) and Han and Kim (2009). Models of saccade programming must take into account signal timing, signal strength, and cognitivecontrol factors in order to accurately model the deployment of the eyes in a visual search task.

In contrast to the independent horse-race model, the competitive integration model does take stimulus spacing into account. Although the relative spacing of the onset and the color singleton did not affect capture rates, spacing did affect saccade latencies, particularly in regard to latencies to an onset or color singleton. For the memorized-color group, not only were latencies to the onset and target faster when they were combined rather than occurring alone (which is also supported by the horse-race model), but the distance of the memorized color from the onset affected saccadic RTs to the onset. In this case, as the distance increased, saccadic RTs to the onset slowed, with the largest distance producing saccade latencies that were slower than those when the onset occurred alone. This directly supports competitive integration and not the horse-race model. However, the onset in this condition did not affect saccades to the memorized color. This asymmetry suggests that memorizing a color can produce a signal that not only can affect signals generated by onsets but also is impervious to the influence of onsets. Similar findings were found for the unique-color group, but in this case the distance between the onset and the unique color singleton affected saccade latencies to both the onset and the uniquecolor singleton, but this effect was limited to a shorter distance. This suggests that holding a color in working memory increases the strength of the signal generated by a matching color singleton. In the case of latencies to the target, neither the singleton nor the onset affected saccadic reaction times, but as with the lack of an effect on capture, this might be due to the slower generation of target-guided signals.

The strength of the memory-driven signal can be attributed to the active rehearsal process of working memory. The memorized color was seen first in the memory display and was relevant to the memory task; only several seconds had passed until that color was seen again in the search display. The active process of memory rehearsal was able to affect the saccade map, and even though the memorized color was search-task irrelevant, the appearance of the stimulus still triggered an orienting response. One possible mechanism is that as the color is rehearsed, reentrant processes could bias early vision, leading to increased saliency for the rehearsed color, in turn leading to increased capture. Interestingly, while memory influenced search, the presence of the memorized color in the search display did not influence memory performance. However, this is consistent with previous results, showing that people have a poor memory for the identity of items they have rejected during search (Beck, Peterson, \& Vomela, 2006).

Author Note The authors thank our lab members for helpful comments on the manuscript. This work was adapted from a portion of J.H.W.'s doctoral dissertation at George Mason University and was funded by a Department of Defense SMART scholarship to J.H.W.

\section{References}

Beck, M. R., Peterson, M. S., \& Vomela, M. (2006). Memory for where, but not what, is used during visual search. Journal of Experimental Psychology. Human Perception and Performance, $32,235-250$. 
Chen, X., \& Zelinsky, G. J. (2006). Real-world visual search is dominated by top-down guidance. Vision Research, 46, 4118-4133.

Coren, S., \& Hoenig, P. (1972). Effect of non-target stimuli upon length of voluntary saccades. Perceptual and Motor Skills, 34, 449-508.

Findlay, J. M. (1982). Global visual processing for saccadic eye movements. Vision Research, 22, 1033-1045.

Folk, C. L., Remington, R. W., \& Johnston, J. C. (1992). Involuntary covert orienting is contingent on attentional control settings. Journal of Experimental Psychology. Human Perception and Performance, 18, 1030-1044.

Godijn, R., \& Theeuwes, J. (2002). Programming of endogenous and exogenous saccades: Evidence for a competitive integration model. Journal of Experimental Psychology. Human Perception and Performance, 28, 1039-1054.

Goldman-Rakic, P. S. (1987). Circuitry of the frontal association cortex and its relevance to dementia. Archives of Gerontology and Geriatrics, 6, 299-309.

Han, S. W., \& Kim, M. S. (2009). Do the contents of working memory capture attention? Yes, but cognitive control matters. Journal of Experimental Psychology. Human Perception and Performance, 35, 1292-1302.

Houtkamp, R., \& Roelfsema, P. R. (2006). The effect of items in working memory on the deployment of attention and the eyes during visual search. Journal of Experimental Psychology. Human Perception and Performance, 32, 423-442.

Kirchner, H., Barbeau, E. J., Thorpe, S. J., Régis, J., \& LiégeoisChauvel, C. (2009). Ultra-rapid sensory responses in the human frontal eye field region. The Journal of Neuroscience, 29, 75997606.

Ludwig, C. J., \& Gilchrist, I. D. (2003). Goal-driven modulation of oculomotor capture. Perception \& Psychophysics, 65, 12431251.

Mannan, S. K., Kennard, C., Potter, D., Pan, Y., \& Soto, D. (2010). Early oculomotor capture by new onsets driven by the contents of working memory. Vision Research, 50, 1590-1597.
Martin-Emerson, R., \& Kramer, A. F. (1997). Offset transients modulate attentional capture by sudden onsets. Perception \& Psychophysics, 59, 739-751.

Miller, J. O. (1986). Time course of coactivation in bimodal divided attention. Perception \& Psychophysics, 40, 331-343.

Olivers, C. N. L., Meijer, F., \& Theeuwes, J. (2006). Feature-based memory-driven attentional capture: Visual working memory content affects visual attention. Journal of Experimental Psychology. Human Perception and Performance, 32, 1243-1265.

Soto, D., Heinke, D., Humphreys, G. W., \& Blanco, M. J. (2005). Early, involuntary top-down guidance of attention from working memory. Journal of Experimental Psychology. Human Perception and Performance, 31, 248-261.

Theeuwes, J. (2010). Top-down and bottom-up control of visual selection. Acta Psychologica, 123, 77-99.

Theeuwes, J., Kramer, A. F., Hahn, S., \& Irwin, D. (1998). Our eyes do not always go where we want them to go: Capture of the eyes by new objects. Psychological Science, 9, 379-385.

Theeuwes, J., Kramer, A. F., Hahn, S., Irwin, D. E., \& Zelinsky, G. J. (1999). Influence of attentional capture on oculomotor control. Journal of Experimental Psychology. Human Perception and Performance, 25, 1595-1608.

Trappenberg, T. P., Dorris, M. C., Munoz, D. P., \& Klein, R. M. (2001). A model of saccade initiation based on the competitive integration of exogenous and endogenous signals in the superior colliculus. Journal of Cognitive Neuroscience, 13, 256-271.

van Zoest, W., Donk, M., \& Theeuwes, J. (2004). The role of stimulus-driven and goal-driven control in saccadic visual selection. Journal of Experimental Psychology. Human Perception and Performance, 30, 746-759.

Woodman, G. F., \& Luck, S. J. (2007). Do the contents of visual working memory automatically influence attentional selection during visual search? Journal of Experimental Psychology. Human Perception and Performance, 33, 363-377.

Zhang, W., \& Luck, S. J. (2009). Feature-based attention modulates feedforward visual processing. Nature Neuroscience, 12, 24-25. 